\title{
The nuclear region of low luminosity flat radio spectrum sources $\star, \star \star$
}

\section{Emission-line spectra}

\author{
A. C. Gonçalves ${ }^{1,2}$ and M. Serote Roos ${ }^{1}$ \\ 1 Centro de Astronomia e Astrofísica da Universidade de Lisboa, Observatório Astronómico de Lisboa, Tapada da Ajuda, \\ 1349-018 Lisboa, Portugal \\ e-mail: adarbon@oal.ul.pt, serote@oal.ul.pt \\ 2 LUTH, Observatoire de Paris-Meudon, 5 place Jules Janssen, 92195 Meudon Cedex, France
}

Received 8 January 2003 / Accepted 4 September 2003

\begin{abstract}
We report on the spectroscopic study of 19 low luminosity Flat Radio Spectrum (LL FRS) sources selected from Marchã's et al. (1996) 200 mJy sample. In the optical, these objects are mainly dominated by the host galaxy starlight. After correcting the data for this effect, we obtain a new set of spectra clearly displaying weak emission lines; such features carry valuable information concerning the excitation mechanisms at work in the nuclear regions of LL FRS sources. We have used a special routine to model the spectra and assess the intensities and velocities of the emission lines; we have analyzed the results in terms of diagnostic diagrams. Our analysis shows that $79 \%$ of the studied objects harbour a Low Ionization Nuclear Emission-line Region (or LINER) whose contribution was swamped by the host galaxy starlight. The remaining objects display a higher ionization spectrum, more typical of Seyferts; due to the poor quality of the spectra, it was not possible to identify any possible large Balmer components. The fact that we observe a LINER-type spectrum in LL FRS sources supports the idea that some of these objects could be undergoing an ADAF phase; in addition, such a low ionization emission-line spectrum is in agreement with the black hole mass values and sub-Eddington accretion rates published for some FRS sources.
\end{abstract}

Key words. galaxies: active - galaxies: BL Lacertae objects: general - galaxies: general

\section{Introduction}

Low frequency surveys of extragalactic radio sources have observed predominantly extended double-lobed radio-galaxies, which display a steep radio spectrum. Surveys carried out at higher frequencies found a larger number of flat radio spectrum (FRS) sources, i.e. sources displaying hard radio spectra and usually characterized by an unresolved, compact radio-core.

Amongst flat radio spectrum sources, we find BL Lacertae objects (BL Lacs). BL Lacs are a class of Active Galactic Nuclei (AGN) characterized by extreme properties, such as high X-ray and $\gamma$-ray luminosities, relatively high optical and radio polarization, and strong and rapid variability at radio, optical and X-ray wavelengths (e.g. Urry \& Padovani 1995); these objects usually show weak or non-existent optical emission lines.

Send offprint requests to: A. C. Gonçalves, e-mail: anabela.goncalves@obspm.fr

^ Based on observations collected at the Multiple Mirror Telescope on Mt. Hopkins.

$\star \star$ Full Fig. 1 is only available in electronic form at http://www. edpsciences.org
BL Lacs, together with Flat Spectrum Radio Quasars (FSRQs), are called blazars. Blazars exhibit characteristics indicative of relativistic beaming (Padovani \& Urry 1990) and seem to cover a wide range of polarization, strength of the emission lines, and position of the synchrotron component in the spectral energy distribution (SED) (Padovani \& Urry 2001). An important distinction in terms of the SED was introduced in BL Lacs by Padovani \& Giommi (1995); these authors separated the objects emitting most of their synchrotron power at high (UV-to-X-ray) and low (far-IR-to-near-IR) frequencies, introducing the terminology of "high-energy peaked BL Lac" (HBL) and "low-energy peaked BL Lac" (LBL) currently in use. Generally, X-ray selected BL Lacs tend to peak at high energies and display less extreme properties than the radio-selected ones, which peak at low energies. It was suggested (Fossati et al. 1998; Ghisellini et al. 1998) that the spectral properties and the luminosity are anti-correlated, such that high luminosity objects are LBLs and low luminosity objects are HBLs.

In recent years there has been a growing interest in the lower luminosity sources (e.g. Falcke 2001; Rector et al. 1999), as well as in those objects displaying intermediate properties 
between HBLs and LBLs (Bondi et al. 2001). Such objects are included in the $200 \mathrm{mJy}$ sample of FRS sources defined by Marchã et al. (1996).

Marchã's et al. sample of low luminosity, core dominated radio sources was selected with the purpose of finding low luminosity BL Lacs and investigating the differences between such objects and other FRS sources. Included in this sample were a number of galaxies which, although radio selected together with BL Lacs and objects displaying a Seyfert-like emission spectrum, and following exactly the same criteria, are optically very different from them. In fact, they do not show any conspicuous signs of activity, the main contribution to their optical spectra being of stellar origin; such objects overlap with the ones dubbed PEGs (for Passive Elliptical Galaxies) described in Antón (2000). These could be objects related to BL Lac phenomena but observed at larger angles to the jet; it is also possible that some of the galaxies are "hidden" BL Lacs, whose nuclear emission is swamped by the host galaxy starlight (Dennett-Thorpe \& Marchã 2000).

Among the galaxies displaying such characteristics, we have selected 19 sources for our studies; these objects are listed in Table 1 of a companion paper (Serote Roos \& Gonçalves 2003, hereafter Paper I). Our purpose was:

(i) to study the stellar content of LL FRS nuclei and to test stellar population synthesis as a method allowing to reveal any hidden optical emission-line features (these go usually undetected, or are very weak, due to the strong dilution induced by the stellar continuum);

(ii) to investigate the nature of the nuclear emission regions and excitation mechanisms at the origin of the weak emission lines present in the spectra of our objects.

In Paper I, we addressed the first point; we discussed the nuclear stellar populations and host properties of the objects in our sample. In this paper we focus our attention on the nuclear emission-line spectra of LL FRS sources; these spectra are presented in Sect. 2. In Sect. 3 we introduce the line profile fitting method and describe how it has been applied to the data. In Sect. 4 we give some notes on the studied objects. In Sect. 5 we discuss the implications of our findings, and in Sect. 6 we summarize the results and conclusions of this work.

\section{The emission-line spectra}

Even though weak emission lines can be detected in the galaxies belonging to our sample (see Fig. 1 in Paper I), their optical spectra are clearly dominated by the stellar continuum which heavily dilutes the features associated with the active nucleus, thus making it difficult to access their true spectroscopic properties (profile, line width, intensity, etc.). Aware of this problem, we have calculated the stellar contribution to each spectrum and removed it from the data (see Paper I), therefore obtaining a new set of 19 emission-line spectra (these are given in the electronic version of Fig. 1, available at http://www .edpsciences.org); here, a single spectrum is presented as an example.

We observe emission lines in all of the objects in the sample. Although emission lines are generally associated with the presence of an AGN, line emission is also frequently found

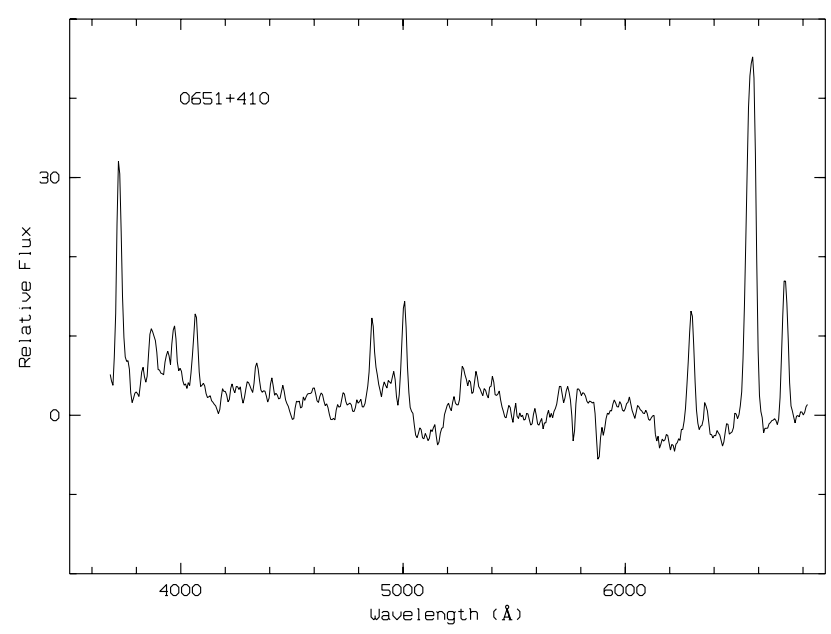

Fig. 1. Example of the rest frame emission-line spectrum obtained for one of the objects in our sample after correction of the stellar contribution; the remaining 18 emission-line spectra are available in electronic form at http: //wWw . edpsciences .org.

in "normal" ellipticals (Goudfrooij 1999) and can be related to phenomena other than an active nucleus, e.g.: photoionization by very hot Wolf-Rayet stars (Warmers), hot $\mathrm{O}$ stars, or old post-AGB stars (Terlevich \& Melnick 1985; Filippenko \& Terlevich 1992; Binette et al. 1994), cooling accretion flows (Heckman 1981; Voit \& Donahue 1997), shock heating through cloud collisions induced by accretion, galaxy interactions, mergers or starburst-driven winds (Fosbury et al. 1978; Fosbury \& Wall 1979; Dopita \& Sutherland 1995, 1996; Alonso-Herrero et al. 2000), etc.

In this study, we favour the AGN hypothesis and interpret the observed weak emission lines as the optical signature of an active nucleus. In support are the AGN-like radio characteristics of our sample, namely a core-dominated emission and a flat radio spectrum, as well as the detection of an X-ray compact core in a few objects. It is intuitive to assume that the same central engine could be at the origin of the observed weak optical emission lines. These lines carry valuable information on the line-emitting regions, ionizing continuum and therefore, on the physical properties of the nuclei. In order to extract these pieces of information from the spectra, a careful modelling of the emission-line features and continuum is required.

\section{Line profile fitting and analysis}

All our emission-line spectra were fitted in terms of Gaussian profiles thanks to a special routine, based on the least squares method, developed by E. J. Zuiderwijk, M.-P. Véron-Cetty and P. Véron. A full description of the method is given in Gonçalves (1999). In the present study, the emission lines $\mathrm{H} \alpha$, [N II] $\lambda$ 6548, 6584, [S II] $\lambda 6716,6731$ and [OI] $\lambda$ 6300, 6363 (or $\mathrm{H} \beta$ and $[\mathrm{O} \mathrm{III}] \lambda 4959,5007$ ) were fitted by one set of seven (or three) Gaussian components; the width and redshift of each component in a set were taken to be the same, as a result of the lines being formed in the same region; this means that, in addition to the line intensities, the free parameters for each set of lines are one width and one redshift. 

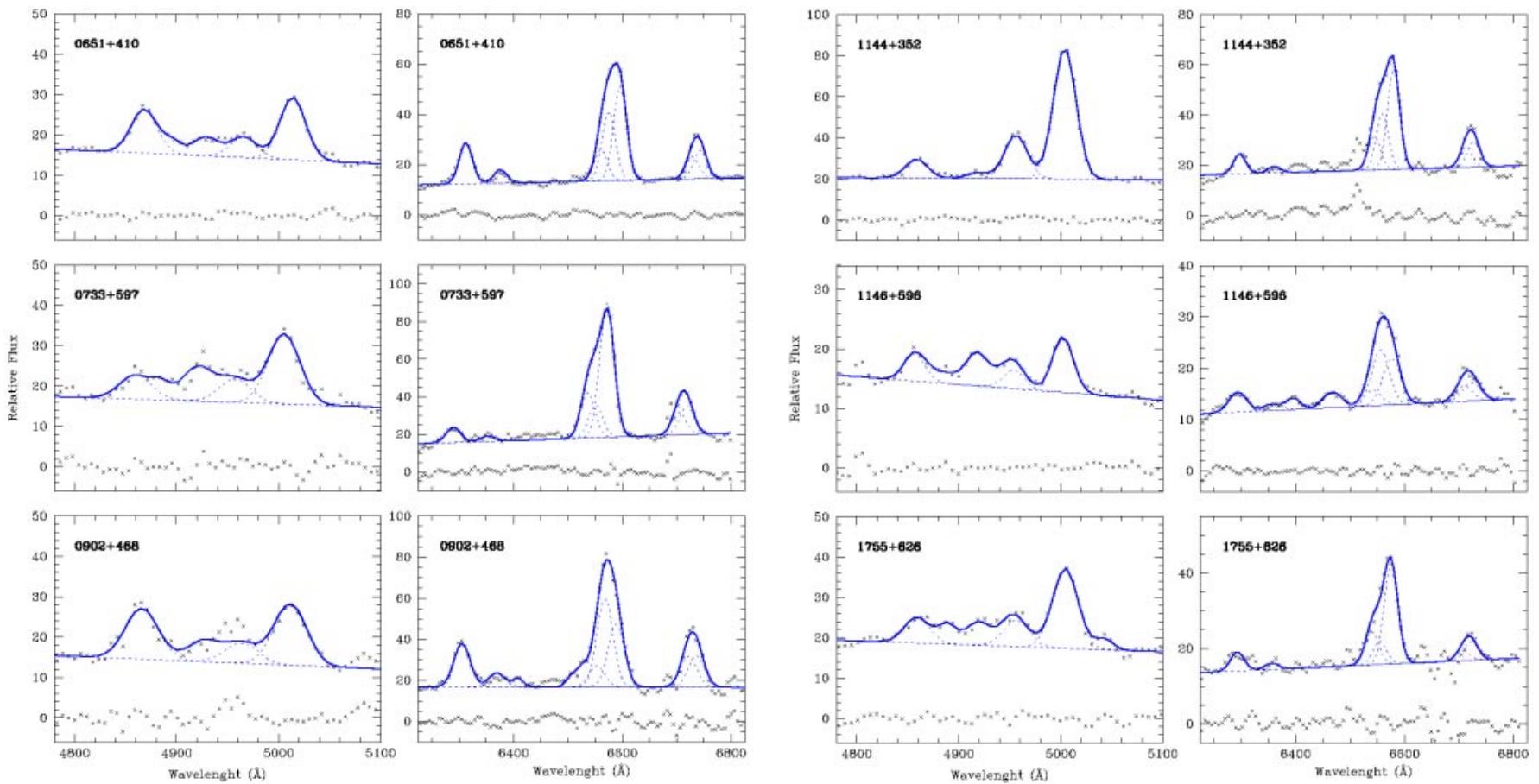

Fig. 2. Best fitting results for six nuclear emission-line regions; for each object, the blue spectral region is shown on the left panel and the red spectral region on the right panel. The data points are represented by crosses; the solid line is the best fit; the dotted lines show the individual components; the lower crosses give the residuals. Both the data and the models were shifted upwards by an arbitrary amount for the sake of clarity. The residuals give an appreciation of the fit quality; in this figure, they are of the same order of magnitude as the noise in the spectra.

The intensity ratios of the $[\mathrm{N}$ II] $] \lambda 548,6584,[\mathrm{O}$ III $] \lambda 4959$, 5007 and $[\mathrm{OI}] \lambda 6300,6363$ lines were set to their theoretical values (3.00, 2.96 and 3.11, respectively; Osterbrock 1974).

The emission lines used in our models were selected in function of their applicability in diagnostic diagrams. By plotting specific line ratios in a 2-dimensional diagram, we are able to identify the type of excitation mechanism at work in the galaxy nucleus; in particular, the use of the Baldwin et al. (1981) or Veilleux \& Osterbrock (1987) diagnostic diagrams allows to classify the nuclear emission-line regions of most galaxies into one of three categories: nuclear H II regions or starbursts, Seyfert 2 galaxies and LINERs. Such diagrams make use of conspicuous optical emission lines, such as $\mathrm{H} \alpha$, $\mathrm{H} \beta,[\mathrm{N}$ II] $\lambda 6584,[\mathrm{O}$ III] $\lambda 5007,[\mathrm{~S}$ II] $\lambda 6717,6713$, [O II] $\lambda 3727$ and $[\mathrm{O} \mathrm{I}] \lambda 6300$.

We have modeled the emission lines as explained above. In a few cases, additional Gaussians were added to fit iron blends, or other weak features present in the spectra; this did not affect the measured intensities of the lines of interest (i.e. the lines used to build the diagnostic diagrams) but contributed to reduce the overall scatter in the fit. Figure 2 shows the best fitting models for six objects in our sample; the parameters resulting from the line profile fitting analysis are given in Table 1.

The line ratios [O III] $] \lambda 5007 / \mathrm{H} \beta$, [N II] $] \lambda 6584 / \mathrm{H} \alpha$ and [O I] $\lambda 65300 / \mathrm{H} \alpha$, used in the Veilleux \& Osterbrock (1987) diagnostic diagrams, take full advantage of the physical distinctions between the various types of objects and minimize the effects of reddening correction and calibration errors. We have calculated these line ratios; the obtained values, given in Table 1, were used to build the diagnostic diagrams shown in Fig. 3. The line-ratios seem to be consistent, providing the same result on both diagnostic diagrams. According to these diagrams, $79 \%(15 / 19)$ of the objects harbour a LINER; the remaining nuclei $(0035+227,0116+319,0848+686$ and $1144+352$ ) display a higher-ionization, Seyfert-like spectrum.

In this study, the mean error in the measurement of the [N II] and [O III] lines intensity is of the order of $13 \%$, in the $\mathrm{H} \beta$ and [OI] lines it goes up to $\sim 24 \%$ and in the $\mathrm{H} \alpha$ line it is $\sim 19 \%$. Usually, no error bars are represented in diagnostic diagrams; however, an estimation of the errors can be calculated based on the previously given values, being of the order of 0.10 and 0.13 in the $x$-axis $(\log [\mathrm{N} \mathrm{II}] \lambda 6584 / \mathrm{H} \alpha$ and $\log [\mathrm{OI}] \lambda 65300 / \mathrm{H} \alpha$, respectively) and 0.11 in the $y$-axis $(\log [\mathrm{O}$ III $] \lambda 5007 / \mathrm{H} \beta)$ in Fig. 3. The classification is therefore robust.

\section{Notes on individual objects}

$\mathbf{0 0 3 5}+\mathbf{2 2 7}$ is a radio-source detected at $5 \mathrm{GHz}$ (Griffith et al. 1990); it has been studied at higher frequencies by Dennett-Thorpe \& Marchã (2000) and more recently at $1.4 \mathrm{GHz}$ (Dennett-Thorpe, private communication). This source has a steep spectral index between 8 and $43 \mathrm{GHz}\left(\alpha_{43}^{8}\right)$ and a relatively low radio polarization; this suggests that the object is not highly boosted, as we would expect from a BL Lac. Following Dennett-Thorpe \& Marchã (2000), this could be a Compact Symmetric Object (CSO). Our spectroscopic analysis points towards a nuclear region with characteristics reminiscent of those of Seyfert 2s; such an optical spectrum is more common amongst double-lobed, FR II radio-galaxies. 
Table 1. Results of the line profile fitting analysis. Column 1 gives the name of the object, Col. 2 the adopted redshift, Cols. 3 and 6 the velocities for each set of components measured on the blue and red spectra, respectively, and de-redshifted using the redshift given in Col. 2; Cols. 4 and 7 the corresponding $F W H M$ (uncorrected for the instrumental broadening), Cols. 5, 8 and 9 the intensity ratios [O III] $\lambda 5007 / \mathrm{H} \beta$, $[\mathrm{N} \mathrm{II}] \lambda 6584 / \mathrm{H} \alpha$ and $[\mathrm{O} \mathrm{I}] \lambda 6300 / \mathrm{H} \alpha$, respectively; n.d. means we could not detect one of the lines (intensity $\sim 0$ ). In Col. 10 we give the spectroscopic classification of the nuclear emission-line region resulting from the diagnostic diagrams.

\begin{tabular}{|c|c|c|c|c|c|c|c|c|c|}
\hline Name & $\bar{z}$ & $\begin{array}{c}V \\
\left(\mathrm{~km} \mathrm{~s}^{-1}\right)\end{array}$ & $\begin{array}{l}F W H M \\
\left(\mathrm{~km} \mathrm{~s}^{-1}\right)\end{array}$ & $\frac{[\mathrm{O} \mathrm{III}]}{\mathrm{H} \beta}$ & $\begin{array}{c}V \\
\left(\mathrm{~km} \mathrm{~s}^{-1}\right)\end{array}$ & $\begin{array}{l}F W H M \\
\left(\mathrm{~km} \mathrm{~s}^{-1}\right)\end{array}$ & $\frac{[\mathrm{N} \text { II }]}{\mathrm{H} \alpha}$ & $\frac{[\mathrm{O} \mathrm{I}]}{\mathrm{H} \alpha}$ & Class. \\
\hline $0035+227$ & 0.097 & -200 & 1078 & 6.09 & -998 & 976 & 1.87 & 0.17 & Seyf 2 \\
\hline $0055+300$ & 0.017 & -111 & 908 & 1.53 & 24 & 1285 & 1.20 & 0.19 & LINER \\
\hline $0116+319$ & 0.060 & -682 & 1506 & 8.22 & -945 & 887 & 1.47 & 0.28 & Seyf 2 \\
\hline $0149+710$ & 0.023 & 243 & 1463 & 0.85 & 786 & 1042 & 1.17 & 1.18 & LINER \\
\hline $0651+410$ & 0.022 & 408 & 1846 & 1.40 & 552 & 1290 & 1.36 & 0.59 & LINER \\
\hline $0729+562$ & 0.107 & 336 & 1728 & 2.60 & 663 & 782 & 1.31 & 0.20 & LINER \\
\hline $0733+597$ & 0.041 & -101 & 2273 & 2.84 & -519 & 1432 & 3.53 & 0.45 & LINER \\
\hline $0848+686$ & 0.041 & -1004 & 1744 & $\gtrsim 10$. & -1242 & 1490 & 1.38 & 0.42 & Seyf 2 \\
\hline $0902+468$ & 0.085 & 70 & 2327 & 1.19 & 225 & 1584 & 0.71 & 0.49 & LINER \\
\hline $1144+352$ & 0.064 & -189 & 1588 & 7.06 & -189 & 1200 & 1.81 & 0.36 & Seyf 2 \\
\hline $1146+596$ & 0.011 & -293 & 1476 & 2.03 & -331 & 1731 & 0.84 & 0.36 & LINER \\
\hline $1241+735$ & 0.075 & 291 & 1455 & 1.01 & 1059 & 1192 & 1.13 & 0.41 & LINER \\
\hline $1245+676$ & 0.107 & 741 & 2000 & 3.51 & 462 & 1036 & 1.99 & n.d. & LINER? \\
\hline $1558+595$ & 0.060 & -498 & 1606 & 2.40 & -751 & 1496 & 1.18 & 0.53 & LINER \\
\hline $1658+302$ & 0.036 & 549 & 1664 & 0.42 & 1203 & 958 & 4.91 & 1.59 & LINER \\
\hline $1703+223$ & 0.050 & -877 & 1875 & 0.88 & -1418 & 915 & 1.27 & 0.48 & LINER \\
\hline $1755+626$ & 0.028 & -153 & 1795 & 3.04 & -386 & 1346 & 4.29 & 0.81 & LINER \\
\hline $2202+363$ & 0.075 & 795 & 3027 & 1.79 & 921 & 1913 & 1.33 & 1.35 & LINER \\
\hline $2320+203$ & 0.039 & 228 & 2017 & 1.86 & 678 & 1067 & 1.79 & 0.28 & LINER \\
\hline
\end{tabular}

$\mathbf{0 0 5 5}+\mathbf{3 0 0}$ is an FR I radio-galaxy with an asymmetric twosided jet and a prominent core; its optical counterpart is the cD galaxy NGC 315, at $z=0.017$. This source has been extensively studied using the VLA and VLBI (e.g. Venturi et al. 1993; Cotton et al. 1999; Dennett-Thorpe \& Marchã 2000) and has been detected at X-ray (Worrall \& Birkinshaw 2000; Terashima et al. 2002) and infrared wavelengths (Golombek et al. 1988). Spectroscopic observations by Ho et al. (1995) suggest the presence of a LINER with a weak broad $\mathrm{H} \alpha$ component. We confirm the low ionization nature of this object, but were not able to detect any broad Balmer line; however, such a component cannot be excluded by our data. The detection of a broad component would be in agreement with the other AGN-like characteristics of the object, namely the presence of a radio jet and a compact, unresolved, X-ray core. Although a small-scale cooling flow may be present in NGC 315 (Worrall \& Birkinshaw 2000), we interpret the observed low ionization spectrum as the optical signature of a low luminosity AGN.

$\mathbf{0 1 1 6}+\mathbf{3 1 9}$ or $4 \mathrm{C} 31.04$ is a variable radio-source with a double-lobed radio structure; it shows the presence of a complex HI absorption (Conway 1996), which hides one of the mini-lobes and partially covers the other. The core source was identified with a faint component between the two extended lobes; no jet-like structures were observed either side of the core (Giovannini et al. 2001). From the steep $\alpha_{43}^{8}$ value, Dennett-Thorpe \& Marchã (2000) suggest that 4C 31.04 is not highly boosted; also its symmetric structure and core-tototal radio power ratio suggest that this source is near the plane of the sky (Giovannini et al. 2001). 4C 31.04 is classified as a low-redshift CSO (Giovannini et al. 2001; Cotton et al. 1995). This object is associated with a galaxy pair (MCG 5-4-17 and 5-4-18) at $z=0.060$. Spectroscopic observations by Gelderman \& Whittle (1994) reveal the presence of $\mathrm{H} \alpha,[\mathrm{N}$ II] $\lambda 6548,6584$ and [O I] $\lambda 6300$ in emission, although the quality of their spectra does not allow to classify the object easily. Our data seem to indicate the presence of a nuclear emission region with properties similar to those of Seyfert 2 galaxies.

$0149+710$ is a core dominated radio galaxy at $z=0.023$. It has been observed at $1.4 \mathrm{GHz}$ by Lara et al. (2001); its radio structure shows a strong asymmetry, with a wide jet in the W-NW direction and a fainter component in the opposite direction, probably related to the counter-jet. At $5 \mathrm{GHz}$, the VLBA image shows a bright core and a collimated jet (Bondi et al. 2001). Thanks to a relatively high optical polarization $(3.3 \%)$ and a Ca II break contrast smaller than 0.4 , this object has been classified as a BL Lac candidate by Marchã et al. (1996); in addition, it shows a high radio polarization and a flat $8.4-43 \mathrm{GHz}$ radio spectrum. Dennett-Thorpe \& Marchã (2000) suggest this could be a "hidden" BL Lac whose non-thermal continuum is being swamped by starlight. Our spectroscopic analysis, carried on the nuclear data after correction for the stellar contribution, 


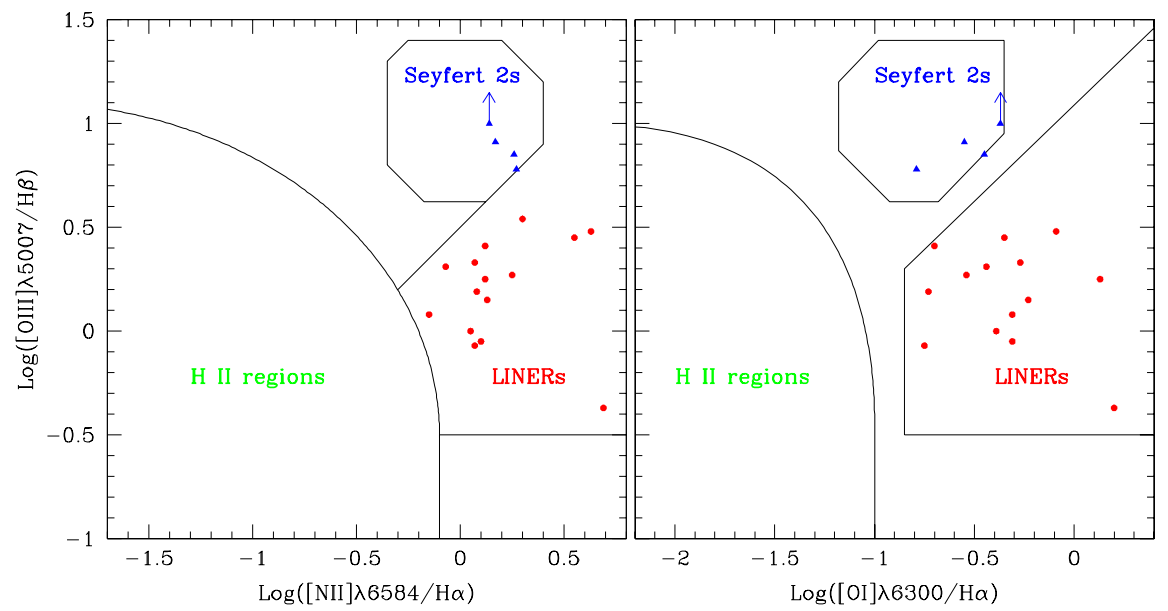

Fig. 3. Diagnostic diagrams showing the logarithm of $[\mathrm{O} \mathrm{III}] \lambda 5007 / \mathrm{H} \beta$ vs. $[\mathrm{N} \mathrm{II}] \lambda 6584 / \mathrm{H} \alpha$ and $[\mathrm{O}$ III $] \lambda 5007 / \mathrm{H} \beta$ vs. [O I] $\lambda 6300 / \mathrm{H} \alpha$. Most of the objects in our sample are located in the LINER region (represented as dots). Only a few nuclei display a higher excitation spectrum, occupying the same location as Seyfert galaxies (represented as triangles). The estimated errors are of the order of 0.10 and 0.13 in the $x$-axis $(\log [\mathrm{N}$ II $] \lambda 6584 / \mathrm{H} \alpha$ and $\log [\mathrm{OI}] \lambda 65300 / \mathrm{H} \alpha$, respectively) and 0.11 in the $y$-axis. reveals a low ionization spectrum typical of LINERs. Detected in the X-rays by ROSAT (Voges et al. 1999), this source is probably a low luminosity AGN observed close to the jet.

$\mathbf{0 6 5 1}+\mathbf{4 1 0}$ is a radio source associated with the elliptical galaxy Zw 204-27. It shows a convex radio spectrum, peaking at frequencies above a few $\mathrm{GHz}$; this source is thus a High Frequency Peaker, or HFP (Dallacasa et al. 2000). However, it has been included by Marecki et al. (1999) in their GPS (GHzPeaked Spectrum) candidate list. This source has a break contrast $\sim 0.47$ and an optical polarization below the $2 \%$ level; due to its borderline properties, Marchã et al. (1996) could not classify this object. $0651+410$ could be a weak BL Lac diluted by the starlight of the host galaxy, as well as a flat radio spectrum galaxy with a naturally low break contrast. Spectroscopic observations by Merighi et al. (1991) reveal the presence of $\mathrm{H} \alpha$, [N II] $\lambda 6548,6584$ and [O I] $\lambda 6300$ in emission. Analysis of our nuclear spectrum, corrected for the host galaxy starlight, shows the presence of a LINER.

$\mathbf{0 7 2 9}+\mathbf{5 6 2}$ is a steep $\alpha_{43}^{8}$ radio source displaying evidence of variability at $1.4 \mathrm{GHz}$ (Dennett-Thorpe \& Marchã 2000); although a number of BL Lacs and BL Lac candidates show variability at radio wavelengths, this source does not seem to be a BL Lac object and remains intriguing. VLBI observations at $5 \mathrm{GHz}$ (Bondi et al. 2001) show that the radio source is rather collimated and presents a series of bright and relatively compact knots; however, the image does not allow us to decide whether the object is one- or two-sided, nor to identify the core; the best candidate, based on observations at $1.6 \mathrm{GHz}$, seems to be the southernmost component. $0729+562$ has been identified with a weak-lined galaxy at $z=0.107$. Our spectroscopic data suggests the presence of a Low Ionization Nuclear Emissionline Region.

$\mathbf{0 7 3 3 + 5 9 7}$ is a compact radio source (Patnaik 1992) identified with an S0 galaxy at $z=0.040538$. Observations with the VLBI (Taylor et al. 1994) revealed a core-jet structure with a faint counter-jet to the south; this source has been included in the extragalactic radio-jets catalogue of Liu \& Zhang (2002). Following Dennett-Thorpe \& Marchã (2000), 0733+597 would constitute a good beamed object candidate. Analysis of our optical data reveals the presence of a LINER, which is most probably associated with a low luminosity AGN. The detection of this source at X-ray wavelengths with ROSAT (Voges et al. 1999) comes in support of this interpretation.

$\mathbf{0 8 4 8}+\mathbf{6 8 6}$ is a radio source with relatively flat $8.4-43 \mathrm{GHz}$ radio spectrum and high radio polarization $(\sim 2.5 \%)$; its optical polarization is lower than $1 \%$. Our spectroscopic analysis, carried out on the nuclear data after correction for the stellar contribution, reveals the presence of a Seyfert-like nuclear emission region. At the present time, we do not dispose of additional pieces of information supporting the presence of an AGN in this object. X-ray observations could contribute to enlighten the situation and provide confirmation of the AGN nature of this source. With a contrast value of 0.46 (Marchã et al. 1996), this could then be another example of a "hidden" BL Lac whose non-thermal continuum is being swamped by the host galaxy starlight. For Caccianiga et al. (2002), however, this is just another Passive Elliptical Galaxy (PEG).

$0902+468$ is a compact radio source identified with a galaxy at $z=0.0848$. Its optical polarization value is less than $1.2 \%$, and the radio polarization is inferior to $0.99 \%$ (Marchã et al. 1996; Dennett-Thorpe \& Marchã 2000). For Caccianiga et al. (2002), this could be a narrow emission-line galaxy; our analysis of the nuclear spectrum, corrected for the stellar contribution, suggests that this object harbours a Low Ionization Nuclear Emission-line Region.

$\mathbf{1 1 4 4}+\mathbf{3 5 2}$ is a low power radio-galaxy showing variable radio polarization at $8 \mathrm{GHz}$ (Dennett-Thorpe \& Marchã 2000); also its arcsecond radio core is variable. This object has been identified with the Zwicky galaxy Zw 186.48 and is a known GPS source (Snellen et al. 1995). 1144+352 was extensively studied by several authors, using different instruments; VLA, MERLIN and VLBI data (Giovannini et al. 1999) show a complex structure over a broad range of physical scales (1 pc-1 Mpc) and confirm previous suggestions of superluminal motion in this source. At parsec resolution, Giovannini et al. (1999) identified the core source and two-sided jets, very asymmetric in shape and properties; from these observations, they computed a jet orientation with respect to the line-of-sight of $25^{\circ}$; this is in agreement with the source properties, intermediate between an FR I galaxy and a BL Lac object. This source was 
considered a BL Lac candidate by Marchã et al. (1996); in addition, it was detected at X-ray wavelengths by ROSAT (Brinkman et al. 1995). All these pieces of evidence suggest that this could, indeed, be a "hidden" BL Lac, whose nonthermal continuum is swamped by starlight, or an object with a jet at larger angles to the line-of-sight. Analysis of the optical spectrum of $1144+352$ suggests the presence of a high ionization nuclear emission region reminiscent of those observed in Seyfert nuclei. This is in agreement with the other AGN-like characteristics of the object.

$\mathbf{1 1 4 6}+596$ is a compact radio source for Condon \& Dressel (1978). VLBI observations at $5 \mathrm{GHz}$ (Wrobel et al. 1985) suggest the presence of three components consistent with an asymmetric core-jet structure; further VLBI imaging of this source provided morphological and spectral evidence leading to different conclusions - namely the presence of twin, parsec-scale jets. The twin-jet kinematics requires that the jets are mildly relativistic and oriented at $\sim 50^{\circ}$ from the line-ofsight (Taylor et al. 1998). 1146+596 has been included in the extragalactic radio-jets catalogue of Liu \& Zhang (2002); the optical counterpart of this source is NGC 3894, a bright galaxy at $z=0.01068$ classified as an elliptical or an S0 (Nilson 1973). Although its optical continuum is dominated by starlight, this galaxy's radio and far-infrared continuum suggest the presence of an active nucleus (Condon \& Broderick 1988). Spectroscopic observations carried by Kim (1989) show the presence of a dust lane and ionized gas along the galaxy's major axis; the gas kinematics are rather peculiar, exhibiting non-circular motions. Analysis of our data shows that the nuclear emission-line region has LINER characteristics; such a low ionization spectrum could be associated with a low power AGN.

$\mathbf{1 2 4 1}+\mathbf{7 3 5}$ is a core-jet radio source (Augusto et al. 1998) identified with a bright galaxy at $z=0.075$. VLBI imaging by Bondi et al. (2001) confirms the compact core and one-sided jet and reveals an unusual radio morphology at larger distances from the core, where the jet starts bending and ends in a compact component. There is no hint of a counter-jet or of any extended emission on the side opposite to the main radio jet. This object shows very high and variable radio polarization and a flat $\alpha_{43}^{8}$ index, which led Dennett-Thorpe \& Marchã (2000) to suggest this could be an active nucleus with a jet close to the lineof-sight. Analysis of our spectroscopic data shows the presence of a Low Ionization Nuclear Emission-line Region, which is compatible with the presence of a low power AGN.

$\mathbf{1 2 4 5 + 6 7 6}$ is a giant radio-galaxy with FR II type morphology (de Vries et al. 1997); it is also a known GPS source. At $1.4 \mathrm{GHz}$, its radio emission is core-dominated (Lara et al. 2001). This source has been identified with the galaxy VII Zw 485 at $z=0.107$. It displays a high contrast value and little radio polarization (Dennett-Thorpe \& Marchã 2000); in consequence, this does not seem to be a good beamed object candidate. Spectroscopic observations published by de Vries et al. (2000) show the presence of $\mathrm{H} \alpha, \mathrm{H} \beta,[\mathrm{N} \mathrm{II}] \lambda 6584$ and [S II] $\lambda 6717,6731$ in emission; our data, corrected for the stellar contribution and modelled with a powerful line-deblending method, confirm these detections as well as the detection of [N II] 6548 in emission. However, the poor signal-to-noise ratio of our spectrum does not allow a firm detection of the [O I] $\lambda$ 6300, 6363 lines; unfortunately, the spectrum published by de Vries et al. was not corrected for the atmospheric absorption, and displays a deep $\mathrm{H}_{2} \mathrm{O}$ absorption at the wavelengths of interest, making it impossible to check for the presence of the [OI] emission lines. Our LINER classification, based on one diagnostic diagram alone, is therefore to be checked through the analysis of better quality data. The presence of an AGN in this source needs still to be confirmed.

$\mathbf{1 5 5 8}+\mathbf{5 9 5}$ is a radio source identified with an elliptical galaxy at $z=0.0602$. It displays a high contrast value and little radio polarization (Dennett-Thorpe \& Marchã 2000); from its steep $\alpha_{43}^{8}$ index, these authors conclude that this is not a highly boosted object and suggest it could be a CSO. VLBA images at $5 \mathrm{GHz}$ show a compact triple-component morphology (Bondi et al. 2001); it is not clear which component is the core. NVSS maps reveal the presence of two slightly resolved radio features, connected by fainter emission, plus another two features to the East, with a two-sided jet visible (Denett-Thorpe, private communication). Spectroscopic observations of this object point towards the presence of a Low Ionization Nuclear Emission-line Region. This is not supported by Caccianiga et al. (2002), who classify $1558+595$ as a simple PEG.

$\mathbf{1 6 5 8 + 3 0 2}$ or 4C 30.31 , is a radio galaxy with FR I type morphology; it shows a $32 \mathrm{kpc}$ one-sided jet embedded in diffuse emission (Gonzalez-Serrano et al. 1993). Capetti et al. (2000) revealed the presence, in this galaxy, of a faint one-sided optical jet, co-spatial with the radio jet. Images obtained at $1.4 \mathrm{GHz}$ show a small double with a compact point source at some distance (Dennett-Thorpe, private communication). This object's radio polarization is below the $2 \%$ level; the optical polarization value is even lower (Dennett-Thorpe \& Marchã 2000; Marchã et al. 1996). 1658+302 does not seem to constitute a good blazar candidate. Analysis of our spectrum suggests the presence of a LINER.

$\mathbf{1 7 0 3}+\mathbf{2 2 3}$ is a radio source associated with an elliptical galaxy at $z=0.04977$. Although its optical polarization level is inferior to $2 \%$, Marchã et al. (1996) have classified this object as a BL Lac candidate based on the contrast value; this has been recalculated by Dennett-Thorpe \& Marchã (2000), whom no longer classify $1703+223$ as a beamed object. Observed at $1.4 \mathrm{GHz}$, this source displays two small compact doubles (Dennett-Thorpe, private communication). Analysis of our spectroscopic data, after correction for the stellar contribution, shows the presence of a Low Ionization Nuclear Emission-line Region.

$\mathbf{1 7 5 5}+\mathbf{6 2 6}$ is a radio source identified with NGC 6521, a galaxy in a cluster. It has a radio polarization value inferior to $2 \%$ and a flat high frequency radio spectrum; based on these characteristics, Dennett-Thorpe \& Marchã (2000) have suggested this could be a "hidden" BL Lac whose thermal continuum is swamped by starlight. Analysis of our optical spectrum, after correction for the stellar contribution, reveals the presence of a LINER; this could be associated with a low power AGN. 
The X-ray detection of this source by Zimmermann et al. (2001) supports this hypothesis. $1755+626$ is thus likely to host a beamed active nucleus.

$\mathbf{2 2 0 2}+\mathbf{3 6 3}$ is a compact radio source identified with a galaxy at $z=0.075$. Unresolved at $1.6 \mathrm{GHz}$, this source has been observed with the VLBA at $5 \mathrm{GHz}$ (Bondi et al. 2001) showing a single component, barely resolved, and no indications of extended emission. This object shows variability at $1.4 \mathrm{GHz}$ (Dennett-Thorpe, private communication); its radio polarization level is inferior to $1.26 \%$, the optical polarization being significatively lower (Marchã et al. 1996; Dennett-Thorpe \& Marchã 2000). Our spectrum shows the presence of a Low Ionization Nuclear Emission-line Region, which could be related to the presence of a low power AGN.

$\mathbf{2 3 2 0}+\mathbf{2 0 3}$ is a compact radio source identified with a galaxy at $z=0.039$. This is another example of an object displaying high radio polarization and a flat $\alpha_{43}^{8}$ index (Dennett-Thorpe \& Marchã 2000). Our spectroscopic analysis reveals the presence of a Low onization Nuclear Emission-line Region; this is most probably associated with a low power AGN. Detection of this object at X-rays wavelengths by Brinkmann et al. (1995) comes in support of this hypothesis. $2320+203$ is thus likely to host an active nucleus observed close to the jet.

\section{Discussion}

\subsection{FRS Sources and low luminosity AGN}

Although usually displaying high luminosities, FRS sources seem to span a large range in luminosity and even jet power (Falcke et al. 2001). In the past few years there has been a growing interest in the low luminosity end of the distribution, with some studies focusing on low power sources (Paper I; Falcke 2001; Falcke et al. 2001; Marchã et al. 1996; Rector et al. 1999). Some of the above mentioned studies were driven by searches for the true nature of blazars and helped to explore the limitations of the present classification.

In low power sources, the emission coming from the host galaxy (hot gas and starlight) is more prone to dilute the nonstellar spectrum, therefore adding to the difficulty in studying the optical properties of the objects. Fully aware of this fact, we have revisited Marchã et al. data in search of additional information on the nuclear region of LL FRS sources. Our results show that the majority of the studied nuclei have LINERtype spectra. It is interesting to note that these low luminosity sources display the same optical properties as other low luminosity AGN (LL AGN), namely a low ionization spectrum.

Is the LINER emission a signature of a low power AGN, or is it being produced by other phenomena (shocks, photoionization by very hot stars, etc.)? The AGN hypothesis is favored in our objects; in support, are the AGN-like radio characteristics of the sources as well as the detection of an X-ray compact core in a few objects. Some of the nuclei show a relatively high degree of radio and optical polarization (see Table 1 of Paper I), which supports their beamed nature. Although most of the sources do not seem to be highly boosted, they can nevertheless be powered by a low luminosity AGN with a jet at larger angles to the line-of-sight.
Our results show that there is a connection between LL FRS sources and low power AGN; other studies give support to our findings. For instance, Nagar et al. $(2000,20)$ and Falcke et al. (2000, 2001) have reported VLA and VLBA observations of a sample of LL AGN selected from the spectroscopic survey of Ho et al. (1995); spectroscopically, most of the objects in this survey are classified as LINERs, with some low luminosity and composite LINER-H II objects also present (Ho et al. 1997). Their observations have revealed that a large fraction of the studied LINERs have flat spectrum compact radio cores, similar to those found in many AGN; in the brightest cores it was even possible to resolve the radio emission into jet-like structures. These authors sustain that at least $50 \%$ of low luminosity AGN in the sample are accretion dominated, with the radio emission presumably coming from the jets and/or an advection-dominated accretion flow. In addition, the brightness temperatures of the radio cores are consistent with the presence of an active nucleus, giving additional support to the AGN-like nature of these LINERs.

Observing the cores of a sample of LL AGN, such as LINERs, these authors found a number of BL Lac-like sources. Our study followed the inverse path: studying a sample of low luminosity FRS sources, known to contain a high fraction of objects with blazar-like properties, we conclude that the large majority display a low ionization spectrum, typical of LINERs.

\subsection{LINERs and ADAFs}

In LL AGN such as LINERs, it has been suggested that radio cores could be due to an advection-dominated accretion flow, or ADAF (Narayan \& Yi 1994, 1995a,b; Fabian \& Rees 1995); an ADAF occurs for small values of the accretion rate (less than $1 \%$ of the Eddington rate).

There are estimations of the BH masses in BL Lacs and radio-loud quasars (e.g. Treves et al. 2002; Falomo et al. 2002; Kotilainen et al. 2002). BL Lac BH masses span a range $5 \times$ $10^{7}-10^{9} M_{\odot}$ (Falomo et al. 2002; Kotilainen et al. 2002); additional results show that the velocity dispersions and $\mathrm{BH}$ masses of BL Lac objects are similar to those obtained for low- $z$ radiogalaxies, in agreement with the unified schemes for BL Lacs and radio-galaxies; also, the average $\mathrm{BH}$ masses in radio-loud quasars are a factor $\sim 2$ greater than those of BL Lacs. The BH masses can be used to derive the Eddington luminosity and this value can be used to calculate the Eddington ratio, provided the total luminosity is known. Treves et al. (2002) estimated the Eddington ratio for the two classes of objects; their values differ by two orders of magnitude in the two classes. This gives support to the hypothesis that the accretion rate changes from largely sub-Eddington in low luminosity, weak-lined sources, to near-Eddington in high luminosity, strong-lined sources.

It has been suggested that the main difference between FR I and FR II radio-galaxies, as well as between LINERs and Seyferts, lies in the accretion rate (for a complete discussion, see the review by Véron-Cetty \& Véron 2000); in the former objects, the accretion rate is small (and so is the $L / L_{\text {Edd }}$ ) and thus, an ADAF forms (Baum et al. 1995). There are evidences for the presence of an ADAF in the radio source Sgr A*, 
which has a LINER spectrum (Yuan et al. 2002) and also in the FR I radio-galaxy M 87 (Reynolds et al. 1996). Moreover, LINERs show characteristics which can be accounted for by the presence of an ADAF, like little short-term X-ray variability, or the presence of double-peaked profiles. Better quality data would allow to search for the possible presence of such double-peaked emission lines in the galaxies of our sample.

An advection-dominated accretion flow could be present in most BL Lacs and BL Lac-type objects. Cao (2002) studied a sample of BL Lac objects and found a bi-modal distribution in their $\mathrm{H} \beta$ luminosity; this author suggests that standard thin disks are associated with sources displaying $L_{\mathrm{H} \beta}>10^{41} \mathrm{erg} \mathrm{s}^{-1}$ and $\mathrm{BH}$ masses in the range $10^{8-10} M_{\odot}$. In lower luminosity sources, like the ones in our sample, he expects the standard thin disk to evolve into an advection-dominated accretion flow, forming a hot and thick disk. The lower limit to the BH mass of these objects is in the range $1.66-24.5 \times 10^{8} M_{\odot}$, in agreement with what is observed in LINERs.

\subsection{The unifying scheme for BL Lacs and FR Is}

Blazars seem to be associated with Fanaroff-Riley type I and II radio-galaxies. According to the unifying scheme, FSRQs and FR II radio-galaxies would be similar objects observed from different angles between the radio jet and the line-of-sight (Barthel 1989), with the former being the beamed version of the latter. In what concerns BL Lacs, there is still some debate on the nature of the parent population; some authors believe it to be the low luminosity, core dominated, FR I galaxies (Urry \& Padovani 1995; Urry et al. 1999, 2000; Kollgaard et al. 1992), while others suggest FR IIs may also contribute (Murphy et al. 1993; Cassaro et al. 1999). A transition population between beamed BL Lacs and unbeamed radio-galaxies has not been detected (Rector et al. 1999); yet, a transition population of low luminosity BL Lacs was predicted to exist in abundance in X-ray selected samples, like the one studied by Browne \& Marchã (1993).

A hot, radiatively inefficient accretion flow such as an ADAF could contribute to suppress the thermal emission in blazar-like objects, therefore allowing for the non-thermal radiation to dominate over larger angles between the jet and the line-of-sight. As a consequence, it would be possible to observe some of the blazars' properties without relativistic beaming taking place. Such objects could be linked to a transition population between beamed BL Lacs and unbeamed FR I galaxies. This could be the case for some of the objects in our sample, e.g. $1146+596$ or $1144+352$.

FR I galaxies usually display an optical LINER spectrum (Laing et al. 1994; Gonçalves 1999) unlike FR IIs, which show nuclear spectra of higher ionization (either Seyfert 1- or Seyfert 2-like). PKS 2014-55 (Jones \& McAdam 1992; Simpson et al. 1996) and S5 2116+81 (Lara et al. 1999) seem to be the exception, displaying simultaneously a FR I radio morphology and a Seyfert-type spectrum. It would be interesting to follow these objects with a long-term spectroscopic program and check for variability in their spectra; detection of a LINER-type spectrum could be an indication that the object has exhausted the gas near the black hole and will undergo an ADAF phase.

\section{Summary and conclusions}

In an effort to better understand low luminosity FRS sources, we undertook the spectroscopic study of 19 objects selected from Marchã's et al. (1996) 200 mJy sample. This sample was expected to contain a high fraction of objects observed at small angles to the jet, like low luminosity BL Lacs and candidate BL Lacs, and includes objects displaying intermediate properties between HBLs and LBLs.

Our study made use of stellar population synthesis as a tool to recover the true spectroscopic characteristics of the nuclei; we then performed a careful analysis of the resulting emissionline spectra. The data were modelled in terms of Gaussian profiles and the measured line ratios were used to identify the excitation mechanisms at work in the nuclear emission-line regions by means of diagnostic diagrams. The results of this analysis show that $79 \%(15 / 19)$ of the objects harbour a Low Ionization Nuclear Emission-line Region, or LINER.

The observed low ionization spectra are interpreted as the optical signature of an active nucleus. Although most of the sources in our sample do not seem to be highly boosted, they can nevertheless be powered by a low power AGN with a jet at larger angles to the line-of-sight. The stellar populations derived in Paper I for these nuclei are in agreement with those usually found in LINERs; also the black hole masses reported for some LL FRS sources are compatible with the presence of a LL AGN, such as a LINER. Our results support a unifying picture involving LL FRS sources and FR I radio-galaxies, which usually display a low ionization optical spectrum. The fact that we observe a LINER-type spectrum in $79 \%$ of the nuclei suggests that the majority of the objects presented here could be undergoing an ADAF phase. The remaining sources may still dispose of enough gas around the black hole to fuel an optically thick, geometrically thin, cool accretion disk; as a consequence, we observe a higher-ionization, Seyfert-like spectrum.

Further observations, carried out at higher spectral resolution and better signal-to-noise ratio, would allow us to better constrain the spectroscopic properties of these nuclei and also to check for variations in their line profiles; it would be particularly interesting to pursue such a study on the few objects displaying radio variability. Finally, better quality data would allow us to search for large Balmer line components and double-peaked emission lines, typical of relativistic accretion disks.

Acknowledgements. We thank S. Antón for fruitful discussions and P. Véron for valuable comments. A.C. Gonçalves and M. Serote Roos acknowledge support from the Fundação para a Ciência e a Tecnologia, Portugal, under grants No. BPD/9422/02 and BPD/5684/2001.

\section{References}

Alonso-Herrero, A., Rieke, M. J., Rieke, G. H., \& Shields, J. C. 2000, ApJ, 530, 688

Antón, S. 2000, Ph.D. Thesis, University of Manchester 
Augusto, P., Wilkinson, P. N., \& Browne, I. W. A. 1998, MNRAS, 299, 1159

Baldwin, J. A., Phillips, M. M., \& Terlevich, R. 1981, PASP, 93, 5

Barthel, P.D. 1989, ApJ, 336, 606

Baum, S., Zirbel, E., \& O’Dea, C. 1995, ApJ, 451, 88

Binette, L., Magris, C. G., Stasinska, G., \& Bruzual, A. G 1994, A\&A, 292, 13

Bondi, M., Marchã, M. J. M., Dallacasa, D., \& Stanghellini, C. 2001, MNRAS, 325, 1109

Brinkmann, W., Siebert, J., Reich, W., et al. 1995, A\&AS, 109, 147

Browne, I. W. A., \& Marchã, M. J. M. 1993, MNRAS, 261, 795

Caccianiga, A., Marchã, M. J., Antón, S., Mack, K.-H., \& Neeser, M. J. 2002, MNRAS, 329, 877

Cao, X. 2002, ApJ, 570, L13

Capetti, A., de Ruiter, H. R., Fanti, R., et al. 2000, A\&A, 362, 871

Cassaro, P., Stanghellini, C., Bondi, M., et al. 1999, A\&AS, 139, 601

Condon, J. J., Huang, Z.-P., Yin, Q. F., \& Thuan, T. X. 1991, ApJ, 378, 65

Condon, J. J., \& Dressel, L. L. 1978, ApJ, 221, 456

Condon, J. J., \& Broderick, J. J. 1988, AJ, 96, 30

Conway, G. M. 1996, Extragalactic radio sources, in proceedings of the 175th Symp. of the International Astronomical Union, held in Bologna, Italy, 10-14 October 1995, ed. R. D. Ekers, C. Fanti, \& L. Padrielli (Kluwer Academic Publishers), 92

Cotton, W. D., Feretti, L., Giovannini, G., et al. 1995, ApJ, 452, 605

Cotton, W. D., Feretti, L., Giovannini, G., Lara, L., \& Venturi, T. 1999, ApJ, 519, 108

Dallacasa, D., Stanghellini, C., Centonza, M., \& Fanti, R. 2000, A\&A, 363, 887

Dennett-Thorpe, J., \& Marchã, M. J. 2000, A\&A, 361, 480

de Vries, W. H., Barthel, P. D., \& O’Dea, C. P. 1997, A\&A, 321, 105

de Vries, W. H., O’Dea, C. P., Barthel, P. D., \& Thompson, D. J. 2000 , A\&AS, 143, 181

Dopita, M. A., \& Sutherland, R. S. 1995, ApJ, 455, 468

Dopita, M. A., \& Sutherland, R. S. 1996, ApJS, 102, 161

Fabian, A. C., \& Rees, M. J. 1995, MNRAS, 277, 55

Falcke, H., Nagar, N. M., Wilson, A. S., \& Ulvestad, J. S. 2000, ApJ, 542, 197

Falcke, H., Markoff, S., \& Biermann, P. L. 2001, Blazar Demographics and Physics, ASP Conf. Ser., 227, 56

Falcke, H. 2001, RvMA, 14, 15

Falomo, R., Kotilainen, J. K., \& Treves, A. 2002, ApJ, 569, 35

Filippenko, A. V., \& Terlevich, R. 1992, ApJ, 397, L79

Fosbury, R. A. E., Mebold, U., Goss, W. M., \& Dopita, M. A. 1978, MNRAS, 183, 549

Fosbury, R. A. E., \& Wall, J. V. 1979, MNRAS, 189, 79

Fossati, G., Maraschi, L., Celotti, A., Comastri, A., \& Ghisellini, G. 1998, MNRAS, 299, 433

Gelderman, R., \& Whittle, M. 1994, ApJS, 91, 491

Ghisellini, G., Celotti, A., Fossati, G., Maraschi, L., \& Comastri, A. 1998, MNRAS, 301, 451

Giovannini, G., Taylor, G. B., Arbizzani, E., et al. 1999, ApJ, 522, 101

Giovannini, G., Cotton, W. D., Feretti, L., Lara, L., \& Venturi, T. 2001, ApJ, 552, 508

Golombek, D., Miley, G. K., \& Neugebauer, G. 1988, AJ, 95, 26

Gonçalves, A.C. 1999, Ph.D. Thesis, University of Provence (Aix-Marseille I)

Gonzalez-Serrano, J. I., Carballo, R., \& Perez-Fournon, I. 1993, AJ, 105,1710

Griffith, M., Langston, G., Heflin, M., et al. 1990, ApJS, 74, 129

Heckman, T. M. 1981, ApJ, 250, L59

Ho, L., Filippenko, A., \& Sargent, W. 1995, ApJS, 98, 477

Ho, L., Filippenko, A., \& Sargent, W. 1997, ApJS, 112, 315
Jones, P. A., \& McAdam, W. B. 1992, ApJS, 80, 137

Kim, D. 1989, ApJ, 346, 653

Kollgaard, R. I., Wardle, J.F.C., Roberts, D.H., \& Gabuzda, D.C. 1992, AJ, 104, 1687

Kotilainen, J. K., Falomo, R., \& Treves, A. 2002, in Galaxy evolution, theory and observations, Cozumel April 8-12, 2002

Laing, R., Jenkins, C., Wall, J., \& Unger, S. 1994, ASP Conf. Ser., 54, 201

Lara, L., Márquez, I., Cotton, et al. 1999, NewAR, 43, 643

Lara, L., Cotton, W. D., Feretti, L., et al. 2001, A\&A, 370, 409

Liu, F. K., \& Zhang, Y. H. 2002, A\&A, 381, 757

Marchã, M., Browne, I., Impey, C., \& Smith, P. 1996, MNRAS, 281, 425

Marecki, A., Falcke, H., Niezgoda, J., et al. 1999, A\&AS, 135, 273

Merighi, R., Basso, L., Vigotti, M., Lahulla, J. F., \& Lopez-Arroyo, M. 1991, A\&AS, 89, 225

Murphy, D. W., Browne, I. W. A., \& Perley, R. A. 1993, MNRAS, 264, 298

Nagar, N. M., Falcke, H., Wilson, A. S., \& Ho, L.C. 2000, ApJ, 542, 186

Nagar, N. M., Falcke, H., Wilson, A. S., \& H., L.C. 2002, NAsRev, 46, 225

Narayan, R., \& Yi, I. 1994, ApJ, 428, L13

Narayan, R., \& Yi, I. 1995a, ApJ, 444, 231

Narayan, R., \& Yi, I. 1995b, ApJ, 452, 710

Nilson, P. 1973, Nova Acta Regiae Soc. Sci. Upsaliensis Ser. V

Osterbrock, D. 1974, Astrophysics of gaseous nebulae

Padovani, P., \& Urry, C. M. 1990, ApJ, 356, 75

Padovani, P., \& Urry, C. M. 2001, Blazar Demographics and Physics, ASP Conf. Ser., 227, 3

Padovani, P., \& Giommi, P. 1995, ApJ, 444, 567

Patnaik, A. R., Browne, I. W. A., Wilkinson, P. N., \& Wrobel, J. M. 1992, MNRAS, 254, 655

Rector, T. A., Stocke, J. T., \& Perlman, E. S. 1999, ApJ, 516, 145

Reynolds, C., Di Matteo, T., Fabian, A., Hwang, U., \& Canizares, C. 1996, MNRAS, 283, L111

Serote Roos, M., \& Gonçalves, A. C. 2003, A\&A, 413, 91 (Paper I)

Simpson, C., Ward, M., Clements, D., \& Rawlings, S. 1996, MNRAS, 281, 509

Snellen, I. A. G., Zhang, M., Schilizzi, R. T., et al. 1995, A\&A, 300, 359

Taylor, G. B., Vermeulen, R. C., Pearson, T. J., et al. 1994, ApJS, 95, 345

Taylor, G. B., Wrobel, J. M., \& Vermeulen, R. C. 1998, ApJ, 498, 619

Terashima, Y., Iyomoto, N., Ho, L.C., \& Ptak, A.F. 2002, ApJS, 139,1

Terlevich, R., \& Melnick, J. 1985, MNRAS, 213, 841

Treves, A., Carangelo, N., Falomo, R., et al. 2002, in Issues in Unification of AGNs, Marciana Marina (Elba island), May 21-24 2001, ed. R. Maiolino, A. Marconi, \& N. Nagar, PASP Conf. Ser., 258,303

Urry, C., Scarpa, R., O’Dowd, et al. 2000, ApJ, 532, 816

Urry, C. M., Falomo, R., Scarpa, R., et al. 1999, ApJ, 512, 88

Urry, C. M., \& Padovani, P. 1995, PASP, 107, 803

Veilleux, S., \& Osterbrock, D. E. 1987, ApJS, 63, 295

Venturi, T., Giovannini, G., Feretti, L., Comoretto, G., \& Wehrle, A. E. 1993, ApJ, 408, 81

Véron-Cetty, M. P., \& Véron, P. 2000, A\&A Rev., 10, 81

Voges, W., Aschenbach, B., Boller, Th., et al. 1999, A\&A, 349, 389

Voit, G. M., \& Donahue, M. 1997, ApJ, 486, 242

Worrall, D. M., \& Birkinshaw, M. 2000, ApJ, 530, 719

Wrobel, J. M., Jones, D. L., \& Shaffer, D. B. 1985, ApJ, 289, 598

Yuan, F., Markoff, S., \& Falcke, H. 2002, A\&A, 383, 854

Zimmermann, H.-U., Boller, T., Döbereiner, S., \& Pietsch, W. 2001, A\&A, 378, 30 
A. C. Gonçalves and M. Serote Roos: The nuclear region of LL FRS sources. II., Online Material p 1

\section{Online Material}


A. C. Gonçalves and M. Serote Roos: The nuclear region of LL FRS sources. II., Online Material p 2
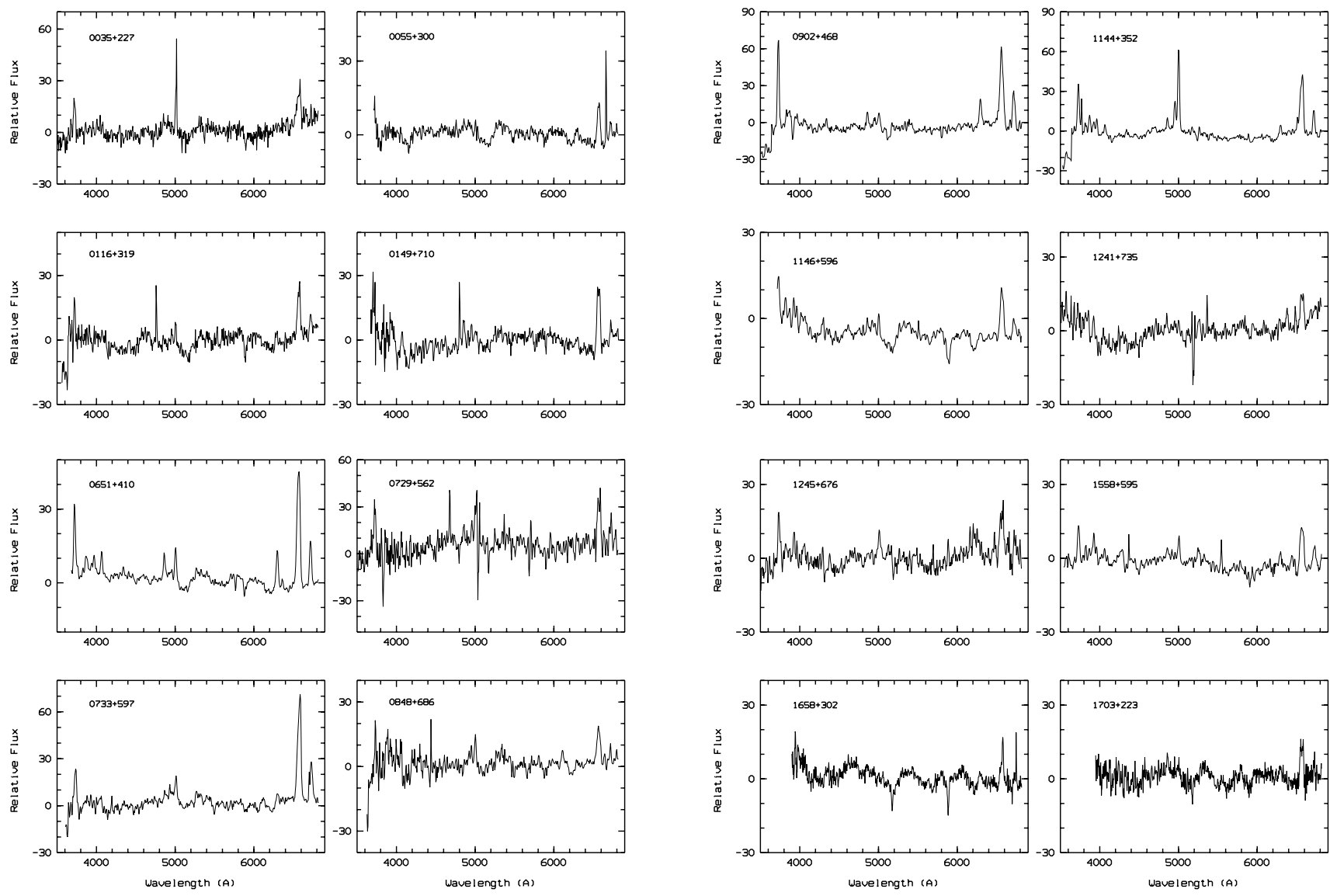

Fig. 1. The rest frame emission-line spectrum obtained for one of the objects in our sample after correction of the stellar contribution.

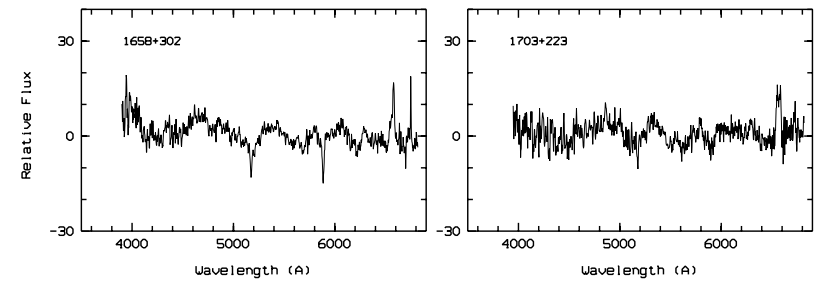

Fig. 1. continued. 
A. C. Gonçalves and M. Serote Roos: The nuclear region of LL FRS sources. II., Online Material p 3
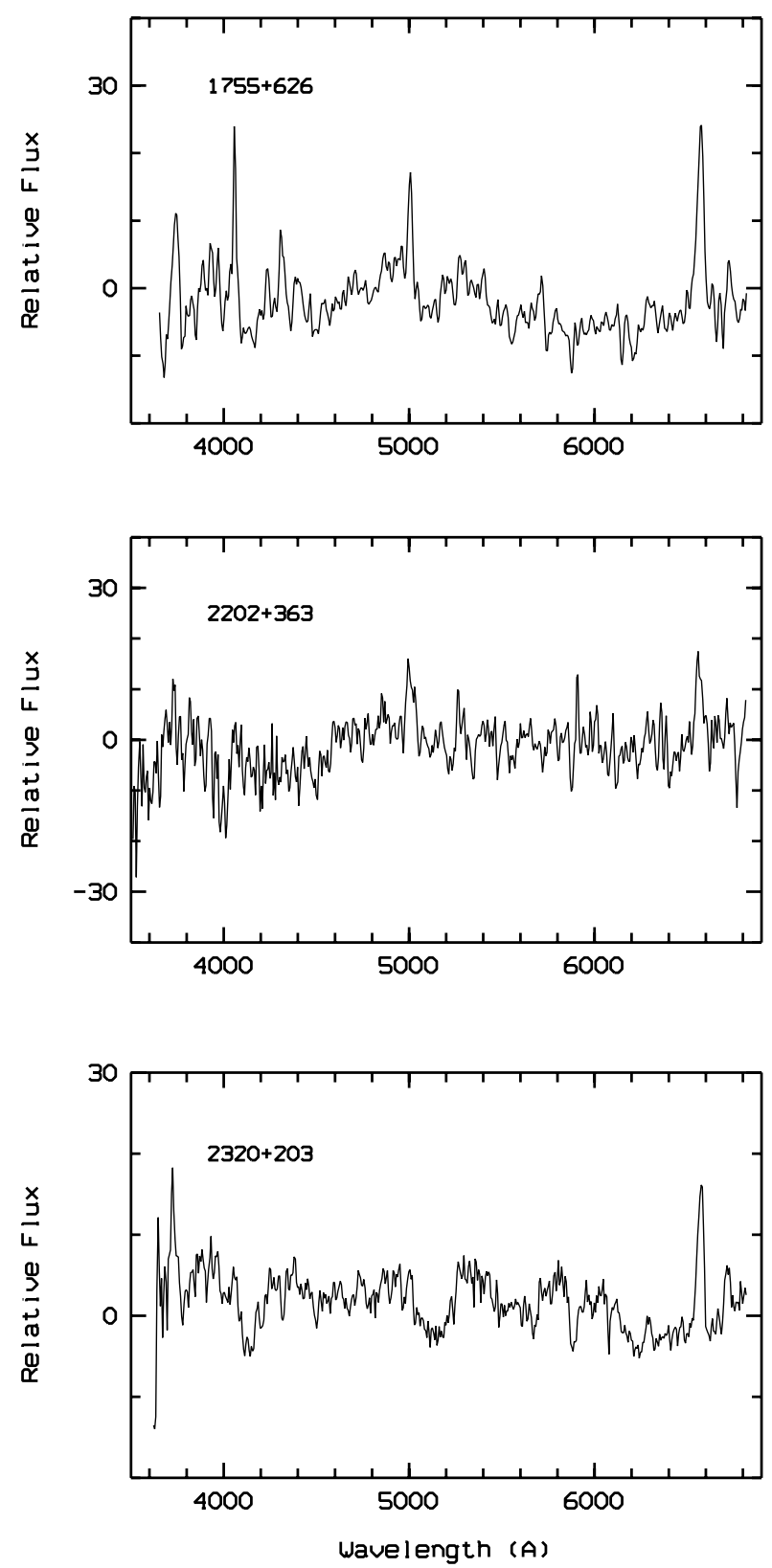

Fig. 1. continued. 